\title{
Silicon sensor technologies for timing
}

\author{
Maria Margherita Obertino* \\ University and INFN Torino \\ E-mail: margherita.obertino@cern.ch
}

The working conditions at future accelerators will require the capability of taking data at unprecedented intensities and the possibility to distinguish events separated by a few tens of picoseconds will become of utmost importance. To face this challenge, intense R\&D programs in silicon sensors are currently being carried on, with the ultimate goal of reaching concurrent excellent position and time resolutions. In this contribution I will review the status and the expectations on the production of fast silicon devices and I will point out the interplay of sensor and electronic parameters in the timing detector design. Two technologies, UFSD and APIX2, will be examined in depth. First results from recently produced prototypes will be shown and compared to simulation.

The 26th International Workshop on Vertex Detectors

10-15 September, 2017

Las Caldas, Asturias, Spain

${ }^{*}$ Speaker. 


\section{Introduction}

Picosecond timing is recently become one of the most important R\&D areas in High Energy Physics (HEP). The addition of track-timing in the event information has been proved to be crucial for the experiments that will run in very dense enviroments like at ones at the future hadron colliders, where high pile-up and high track density will pose severe requirements to both sensors and electronics. For the upgrade of the present LHC detectors in view of the incoming high luminsity run (HL-LHC [1]), two different solutions are being considered. ATLAS and CMS plan to add, for LHC-Run4 ( 2027), a dedicated timing layer ([2],[3]) to measure the time of the reconstructed tracks. LHCb is considering the option to install for the beginning of LHC-Run5 ( 2031) a new Vertex Detector (VELO) [4], based on high-granularity pixels able to measure accurately time and position. The longer timescale and the reduced size of this detector allow for the design of a more complex object which, making available in the pattern recognition both time and space coordinates, allows to reconstruct tracks using only time compatible hits. Radiation hardness up to fluences of the order of $10^{16} \mathrm{n}_{e q} / \mathrm{cm}^{2}$, low material budget and low power consumption are additional mandatory requirements in all the mentioned applications.

Thanks to their high granularity, excellent spatial resolution, low material budget and high radiation tolerance, silicon detectors are the key elements of most of the current HEP tracking systems. Nevertheless, the time capability of these detectors is limited by the signal formation process itself. The state of the art for hybrid silicon pixel detectors designed to measure both time and position of charged particles is represented by the Gigatracker (GTK) of the NA62 experiment [5]. GTK is made of 3 stations instrumented with $200 \mu \mathrm{m}$ thick planar silicon sensors bump-bonded to ten read-out ASIC implemented in $130 \mathrm{~nm}$ CMOS technology. The chip (TDCpix) consists of 40x45 pixels, 300x300 $\mu \mathrm{m}^{2}$ each. The time resolution achieved with this architecture has been measured to be $130 \mathrm{ps}$. This represents the best timing result obtained with silicon detectors on running experiments so far.

In order to improve this result, several R\&D activities are on going. Two of them, UFSD (Ultra Fast Silicon Detector) [6] and APIX2 (Two-tier Avalanche Pixel Sensors) [7], will be discussed in this contribution. The schematic cross section of the sensors proposed by the two projects is shown in Figure 1. Interesting results are also expected by the optimization of 3D sensors for timing

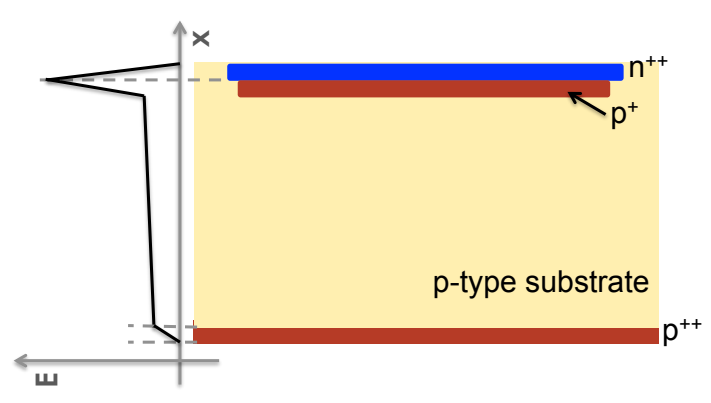

(a) UFSD

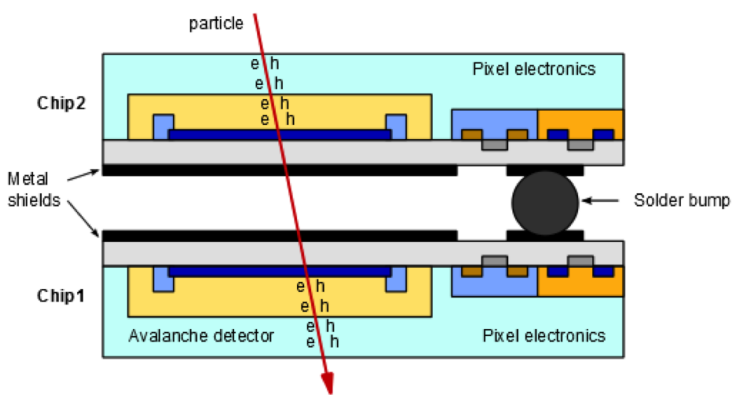

(b) APIX2 [8]

Figure 1: Sensor schematics

measurements [9]. Even if this R\&D is currently less advanced than the two reported hereafter, the 
fast response characteristic of 3Ds and the superior radiation tolerance make these devices strong candidates for a $4 \mathrm{D}$ tracking system able to work in a harsh radiation enviroment. A boost on their development is expected in the next few years.

\section{Time resolution in silicon detectors}

A simplified model of a silicon-sensor-based timing detector [6] consists of a capacitor with a current source in parallel, read out by an amplifier which also shapes the signal. The shaper output, compared to a theshold $\mathrm{v}_{t h}$, determines the particle arrival time that is finally stored in a TDC. In this model, the time resolution of the detector can be parametrized as:

$$
\sigma_{t}^{2}=\sigma_{\text {Jitter }}^{2}+\sigma_{\text {TimeWalk }}^{2}+\sigma_{\text {LandauNoise }}^{2}+\sigma_{\text {Distortion }}^{2}+\sigma_{T D C}^{2}
$$

The contribution of the TDC binning $\left(\sigma_{T D C}=T D C_{b i n} / \sqrt{12}\right)$ is negligible and can be ignored in this discussion. The jitter term is directly proportional to the total noise of the detector and inversely proportional to the slew rate, i.e. the slope of the signal $\mathrm{dV} / \mathrm{dt}$ around the comparator threshold. This term can be reduced by optimizing the sensor and the electronics design concurrently. The Landau fluctuations produce two effects: the Time Walk and the Landau Noise, namely the variation of the signal shape due to the non-homogeneous energy deposition along the track. While the Time Walk effect can be minimized by an opportune read-out electronics, including for example Constant Fraction Discriminators (CFD) or Time over Threshold circuits (ToT), the Landau Noise can be mitigated by the sensor design but cannot be completely cancelled and at the end sets the intrinsic limit of the sensor in measuring time. Finally, $\sigma_{\text {Distortion }}$ comprises the effects on the signal shape of non uniformities of the weighting field and of the carrier's drift velocity inside the sensor volume. This term can be minimized by setting the electric field high enough to saturate the drift velocity everywhere in the sensor and by choosing an appropriate geometry.

The design of the UFSD and APIX2 detectors has been driven by the goal of optimizing the time resolution through the minimization of the discussed contributions.

\section{UFSD: Ultra Fast Silicon Detector}

UFSDs are Low-Gain Avalanche Diodes (LGAD) optimized for timing measurements. LGADs [10] are n-in-p silicon sensors with a thin, highly doped $\mathrm{p}+\operatorname{implant}\left(\mathrm{N}_{A} \sim 10^{16} / \mathrm{cm}^{3}\right)$ just below the $\mathrm{n}+$ electrode. This extra layer, usually referred to as multiplication or gain layer, generates a local electric field high enough $\left(\mathrm{E}_{\text {peak }} \sim 300 \mathrm{kV} / \mathrm{cm}\right.$ ) to produce a controlled avalanche when is crossed by charge carriers. The doping profile of the gain layer is the principal technological parameter to be controlled in oder to set the gain value. Most of the sensors produced so far have Boron as gain layer dopant. Figure 1a shows a schematic cross section of the device.

LGADs were proposed and developed at Centro Nacional de Microelectronica (CNM) in 2010 within a RD50 common project [11]. Since then the interest on this kind of devices is increased and nowadays four manufacturers are involved in their production: CNM in Spain, Fondazione Bruno Kessler (FBK) in Italy, Hamamatsu Photonics (HPK) in Japan and Micron Semiconductors LTD in UK. 
The UFSD choice to optimize LGAD for timing is to produce thin sensors and with low gain. This choice has been made taking into consideration the characteristics of the LGAD current signal, whose rise time is proportional to the sensor thickness while the maximum value is determined by the gain. Gain and small thickness are therefore fundamental ingredients to increase the slew rate. Nevertheless, since gain amplifies also the shot noise, an high gain can degradate the time resolution of irradiated detectors. The lowest gain sufficient to perform accurate time measurements has to be set to operate these sensors in harsh radiation enviroments. From simulation this value has been estimated to be between 10 and 20. In thinner sensors also the effect of Landau Noise is minimized.

\subsection{Time resolution of non-irradiated UFSD sensors}

The time resolution of non-irradiated USFD sensors of different thickness produced by CNM and HPK has been measured in laboratory and in beam tests. The latest results are reported in Figure 2 [12]. The plot shows the gain dependence of the time resolution for $50 \mu \mathrm{m}$ thick, $1.3 \mathrm{~mm}$ single pads produced by HPK, read out by a board developed at the University of California Santa Cruz specifically for UFSDs [13]. Full markes rapresent the time resolution while open markers rapresent the jitter contribution derived from the signal shape. Measurements were performed in laboratory with a ${ }^{90} \mathrm{Sr} \beta$-source.

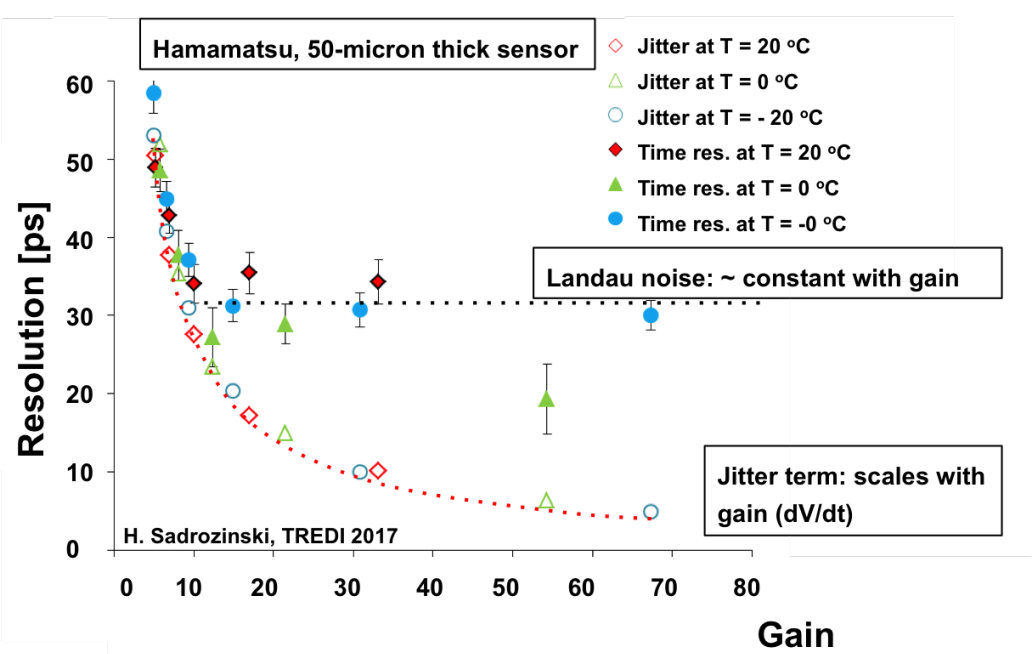

Figure 2: Gain dependence of time resolution (full markers) of $50 \mu \mathrm{m}$ thick UFSD sensors produced by HPK. Measurement were perfomed at three different temperatures. The jitter contribution, derived from the signal shape as $\sigma_{j i t t e r}=\mathrm{N} /(\mathrm{dV} / \mathrm{dt}$ ), is also shown (open markers) [12], [17].

There are several important indications in this plot: (I) the jitter decreases as the gain increases; (II) the time resolution flattens around $30 \mathrm{ps}$ which consequently is the limit set by the Landau Noise in $50 \mu \mathrm{m}$ thick sensors (improvements are expected for thinner sensors); (III) the $30 \mathrm{ps} \mathrm{limit}$ is reached at gain 15, as foreseen by simulations. Compatible results have been obtained with sensors manifactured by CNM; specifically, a time resolution of $27 \mathrm{ps}$ has been measuerd on a test beam for $45 \mu \mathrm{m}$ thick, $1.7 \mathrm{~mm}^{2}$ UFSD pads at a bias voltage of $230 \mathrm{~V}$ [13]. The UFSD planes currently installed close to the LHC beam line in the CT-PPS (CMS-TOTEM Precision Proton Spectrometer) detector [14] are part of the same CNM production. 


\subsection{Effect of radiation on UFSD}

Being UFSDs silicon sensors, their performances are deteriorated by radiation. In intense radiation enviroments the most relevant effects are caused by the damage of the detector bulk due to the non-ionizing energy loss. The three main changes in macroscopic detector properties due bulk damages are the increase of the leakage current, the degradation of charge collecton efficiency and the variation in the effective doping concentration. These effects are proportional to the fluence and depend on the sensor thickness. Among them, the most relevant for thin (50 $\mu \mathrm{m}$ or less) UFSD sensors is the increase of the leakage current generated in the bulk which is amplified by the gain. This effect can be minimized by choosing a low gain value and by cooling the sensors.

In addition to the damages typical of silicon sensors without gain, UFSDs suffer the effects of radiation on the gain layer. Specifically, the doping concentration of the multiplication layer changes through the acceptor removal mechanism: Boron atoms are displaced becoming interstitial and don't contribute to the gain mechanism any more.

In order to study the behaviour of UFSDs in intense radiation enviroments, several CNM and HPK sensors were irradiated with neutrons at the TRIGA reactor in Ljubljana at different fluences between $10^{14} \mathrm{n}_{e q} / \mathrm{cm}^{2}$ and $6 \cdot 10^{15} \mathrm{n}_{e q} / \mathrm{cm}^{2}$. A degradation of the time resolution due to the decreasing value of the gain has been observed, as reported in [15] and [16] for CNM and HPK devices, respectively. Figure 3 [17] shows the measurements performed before and after irradiation on circular $50 \mu \mathrm{m}$ thick HPK LGAD sensors with an active area of $3.1 \mathrm{~mm}^{2}$. Plot 3 a indicates that an higher bias voltage is required to reach a given gain value $\left(\mathrm{V}_{\text {bias }}^{G}\right)$ after irradiation and that $\mathrm{V}_{\text {bias }}^{G}$ increases with fluence. The increment of bias voltage is needed to compensate the drop of doping in the multiplication layer which generates a weaker electric field. The time resolution as a function of the gain for different fluences is shown in Figure 3b. Measurements indicate that $\sigma_{t}$ improves when the gain increases, as expected. For sensors irradiated at $6 \cdot 10^{15} \mathrm{n}_{e q} / \mathrm{cm}^{2}$ a time resolution of about $50 \mathrm{ps}$ is reached at gain $3\left(\mathrm{~V}_{\text {bias }}^{G=3} \sim 700 \mathrm{~V}\right)$ and at a temperature of $-20^{\circ} \mathrm{C}$. The plot also shows that for gain below 10 sensors irradiated at a fluence $\geq 10^{15} \mathrm{n}_{e q} / \mathrm{cm}^{2}$ reach a better time resolution than lower-irradiated sensors. According to simulations this is due to the fact that for the higher-irradiated sensors the applied bias voltage is high enough to create an electric field sufficient to generate multiplication in the bulk and not only in the gain layer.

In order to mitigate the disappearance of the gain caused by radiation damage, two different solutions have been proposed within the RD50 collaboration:

- raplace Boron doping with Gallium which is less prone to become intertitial

- add high level of Carbon which, having an higher probability to become interstitial, protects both Boron and Gallium dopant.

Following these indications, both FBK and CNM have recently produced sensors with different gain layer configurations: Boron, Boron with Carbon, Gallium, Gallium with Carbon.

\subsection{The latest FBK production: $50 \mu \mathrm{m}$ thick sensors with different geometries and gain layer configurations}

FBK has recently manifactured $50 \mu \mathrm{m}$ thick UFSD sensors with 4 different gain layer configurations (Boron, Boron with Carbon, Gallium, Gallium with Carbon) and two Carbon concentrations 


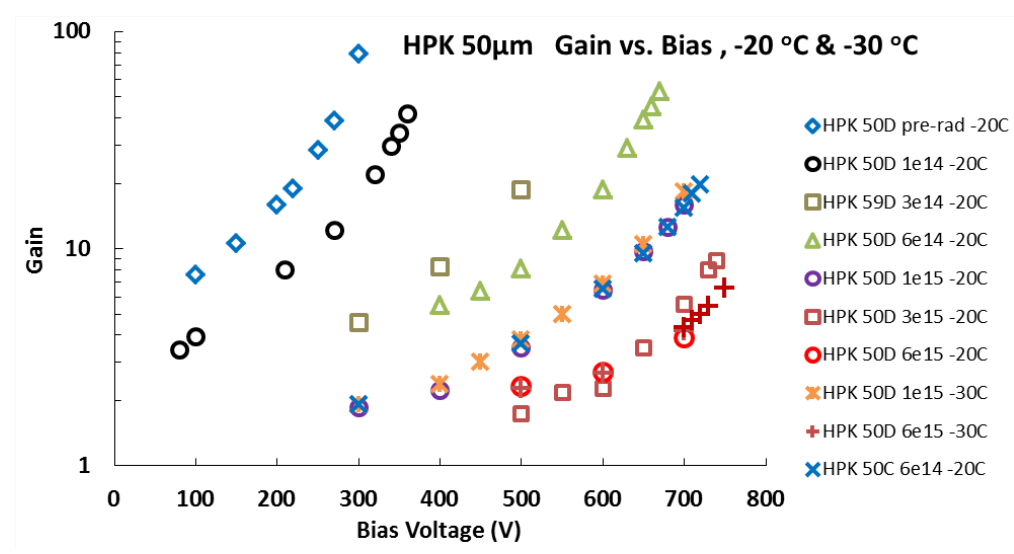

(a) Gain versus bias voltage. Measurements performed at $-20^{\circ} \mathrm{C}$ and $-30^{\circ} \mathrm{C}$

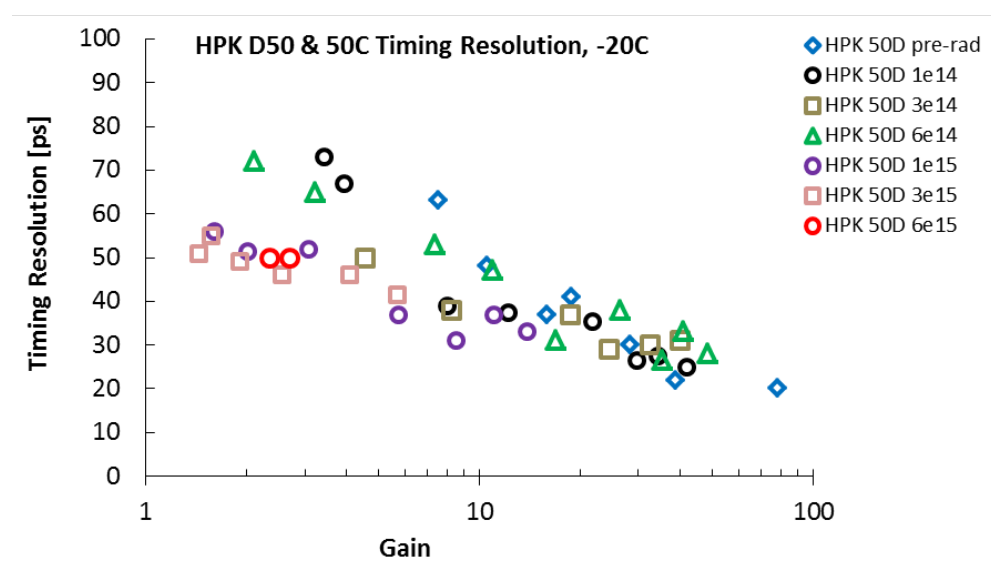

(b) Time resolution versus gain. All measurements performed at $-20^{\circ} \mathrm{C}$

Figure 3: Gain dependence on the bias voltage and time resolution dependence on the gain for HPK UFSD sensors before and after irradiation [17]. Measurement were performed after irradiation at different neutron fluences, as indicated in the legend.

(Low Carbon, High Carbon=10xLowCarbon). For Boron, wafers with two diffusion temperatures (high/low diffusion) have also been produced. Each wafer contains several structures: single pads, multipads, strips with different pitch and lenght, pixel sensors designed to be bump-bonded to the NA62 TDCpix chip.

The production was completed in June 2017. Electrical measurements performed on wafer at FBK showed a very good homogeneity and the expected behavior for all sensors. Figures 4 and 5 show the results of IV and CV measurements for $1 \mathrm{~mm}^{2}$ sensors with different multiplication layer configurations. Sensors with Boron and Gallium implant have similar behaviour. Figure 4 shows that the co-implantation of $\mathrm{C}$ has the effect of increasing the leakage current; however, since the capacitance depenence on the bias voltage is the same in sensor with and without Carbon, the internal electric properties of the devices remain unchanged. The CV curves indicate that in $\mathrm{Ga}$ structures full depletion occurs at an higher bias voltage and that the co-implantation of $\mathrm{C}$ has the effect to lower the gain layer depletion point both in Gallium and Boron. 


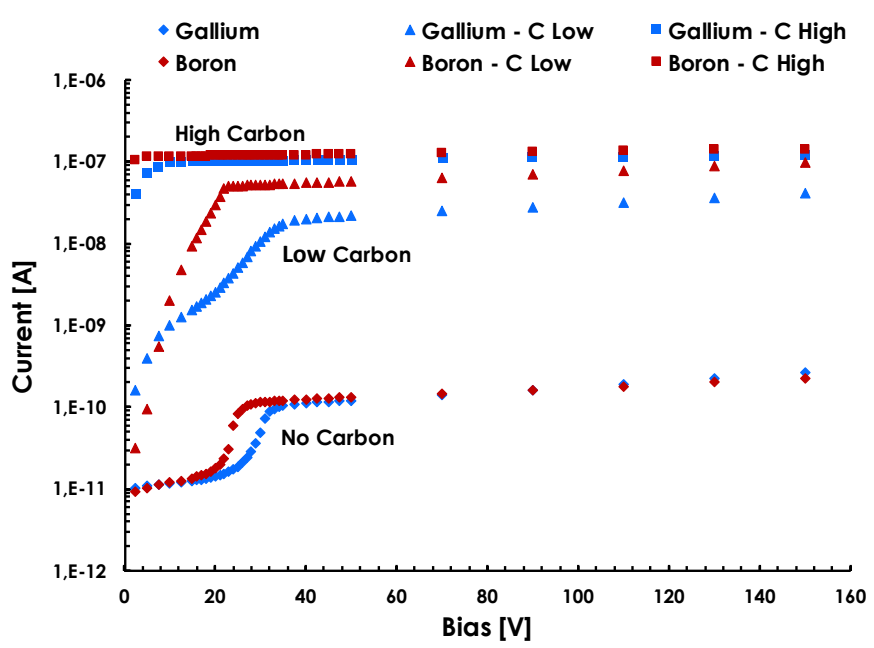

Figure 4: IV curves measured for FBK sensors with different dopant of the gain layer: Boron with and without Carbon, and Gallium with and without Carbon.

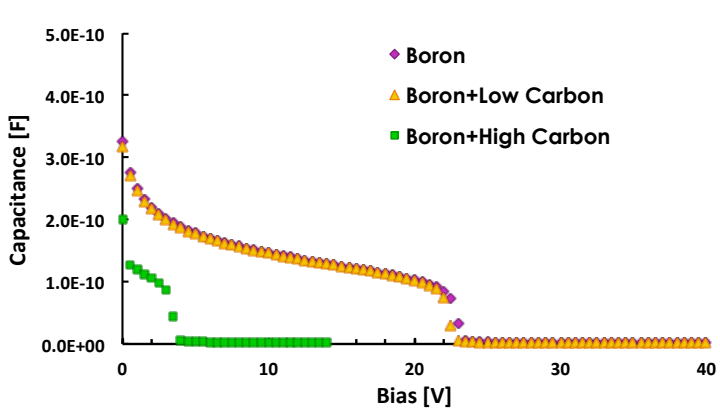

(a) $\mathrm{B}, \mathrm{B}+\mathrm{C}$

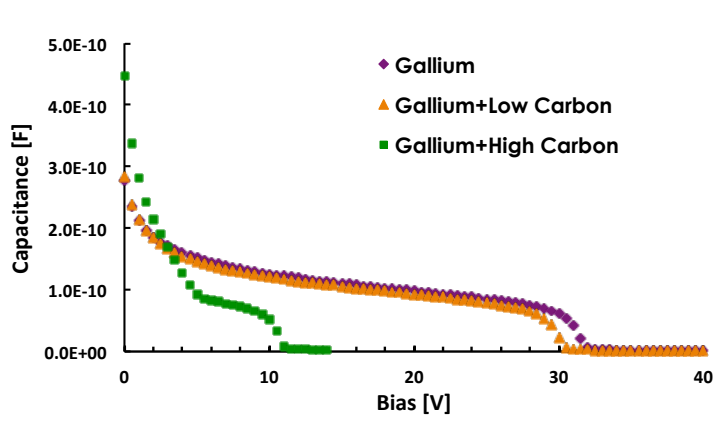

(b) $\mathrm{Ga}, \mathrm{Ga}+\mathrm{C}$

Figure 5: CV curves measured for FBK sensors with different dopant of the gain layer: Boron with and without Carbon (a) and Gallium with and without Carbon (b).

In addition to single pads, also sensors with GTK compatible layout were tested. Specifically, UFSDs were bump-bonded to the TDCpix chip at IZM and tested with beam at CERN. Even if a detailed analysis is still ongoing, preliminary results look promising. The fill factor of these sensors was not optimized $(\sim 70 \%)$. A new desing with a reduced dead area between adjacent pixels is in preparation for the next FBK production.

\section{APIX2: two-tier avalanche pixel sensors}

APIX2 [18] is an INFN project whose purpose is to develope charged particle detectors based on a Geiger-mode avalanche sensors (SPAD) combining fine pixelation, low material budget, low power consumption and good time resolution. It proposes an evolution of the APIX detector concept [19] proposed for the first time in 2012. SPADs are very well established detectors for photons but their use for charged particles requires a strong suppression of the dark count rate (DCR). The 
idea of APIX2 is to use two SPADs in coincidence so that the DCR is reduced by a factor which is proportional to the time resolution of the coincidence.

APIX2 sensors are designed in a commercial $150 \mathrm{~nm}$ CMOS process and the read-out electronics is monolitically integrated. The vertical interconnection between the two SPADs is done via bump-bonding. Two types of Geiger-mode avalanche sensors have been produced based on FBK experience, with the junction implemented as p+ or pwell in a deep nwell (details in [20]). Both sensors have an active thickness below $2 \mu \mathrm{m}$ and are isolated from the substrate by the nwell layer. This makes possible to thin sensors down to few microns without compromising their functionalities. The read-out electronics on each SPAD is composed by a low threshold comparator, which digitizes the signal, followed by a monostable circuit which shortens it. The output of the two monostables is sent to the coincidence circuit located in the bottom pixel, which also includes the digital circuits for data storage and read-out. The coincidence resolution time depends on the monostable pulse width $\Delta \mathrm{T}$, that can be set to three values: $750 \mathrm{ps}, 1.5 \mathrm{~ns}$ or $10 \mathrm{~ns}$.

The sensor prototypes produced for APIX2 consist of 16x48 arrays of 50x75 $\mu \mathrm{m}^{2}$ pixels with active areas of different size, from $30 \times 30$ to $43 \times 45 \mu \mathrm{m}^{2}$. The results presented hereafter are obtained with pixels with the largest active area, which corresponds to a fill factor of $51.6 \%$

\subsection{First experimental results}

A lot of measurements have been done on the produced APIX2 devices, both on single layer and vertically integrated sensors.

Figure 6 shows the dark count rate coincidence (DCRC) for 3 different settings of the monostable, together with the dark count rate of the separate layers $\left(\mathrm{DCR}_{1}\right.$ and $\left.\mathrm{DCR}_{2}\right)$. The average DCRC is $27 \mathrm{~Hz} / \mathrm{mm}^{2}$, about 5 order of magnitudes lower that single SPAD DCR and in good agreement with the theoretical expectation $\mathrm{DCRC}_{\text {exp }}=\mathrm{DCR}_{1} \cdot \mathrm{DCR}_{2} \cdot 2 \Delta \mathrm{T}$.

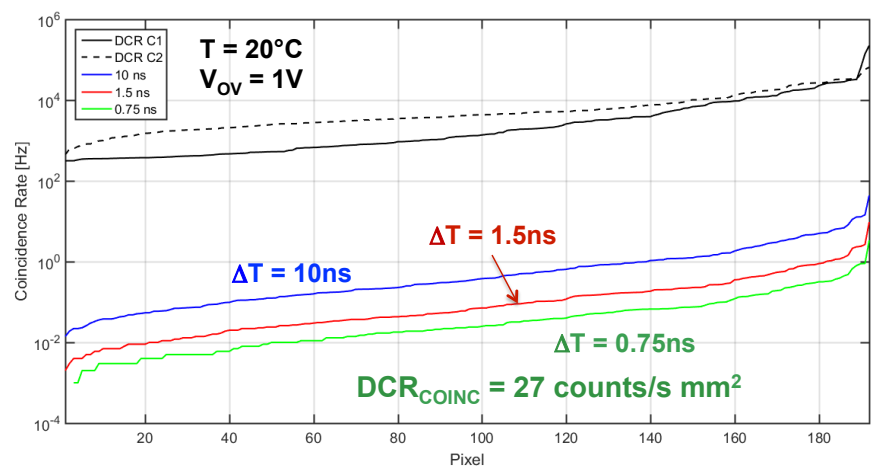

Figure 6: Dark count rate distribution of the APIX2 pixels measured separately for the two sensors and for their coincidence. Measurements done at $\mathrm{T}=20^{\circ} \mathrm{C}$ and overvoltage $\mathrm{V}_{O V}=1 \mathrm{~V}$, for 3 different settings of the pixel monostable circuit $(\Delta \mathrm{T}=750 \mathrm{ps}, 1.5 \mathrm{~ns}, 10 \mathrm{~ns})$ [8].

A validation of the sensor operation was performed using a $\beta$-source with measurements done at $5^{\circ} \mathrm{C}$ to reduce the DCR below the count rate produced by a $\beta$ particle $(\beta-\mathrm{CR})$. The average $\beta$-CR was approximately $33 \mathrm{mHz}$ per pixel which corresponds to $8.8 \mathrm{~Hz} / \mathrm{mm}^{2}$. This value was found in 
good agreement with the expectation derived with a Monte Carlo simulation where the acceptance and all the sources of inefficency were taken into account.

An average optical cross-talk between neighboring pixels of the order of a few percent has been measured at 3V overvoltage [21]. Preliminary measurements, performed with a blue picosecond laser on unshielded single sensors with a diameter of $10 \mu \mathrm{m}$, showed a single-photon time resolution in the order of 60 ps FWHM, even though the electronics was not yet optimized for timing [8]. The measurement was performed also on vertically integrated APIX2 sensors in a beam test; results are expected soon.

\section{Conclusions}

In this paper we have presented the status of two $R \& D$ projects whose aim is to develope high granularity silicon sensors able to accurately measure the time of arrival of a charged particle: UFSD and APIX2.

The development of UFSD sensors and the study of their properties is one of the most important activities of the RD50 collaboration. The behaviour of these devices is well understood and reproduced by simulation. A time resolution of $30 \mathrm{ps}$ has been measured on beam tests for $1 \mathrm{~mm}^{2}$ pads produced by different manufacturers. After exposure to neutron fluence of $6 \cdot 10^{15} \mathrm{n}_{e q} / \mathrm{cm}^{2}$ a $\sigma_{t} \sim$ $50 \mathrm{ps}$ has been observed for sensors cooled at $-20^{\circ} \mathrm{C}$; improvements are expects with new implants of $\mathrm{Ga}$ and $\mathrm{C}$, currently under test. Next productions will include sensors with a reduced cell size and an improved fill factor.

For APIX2, the first demonstration of a pixel array for charged particles based on Geiger-mode avalanche detectors in coincidence has been presented. Experimental results indicate that detectors with a fine pixelation, a good uniformity and small power consumption are feasible with the chosen technology. Further work is foreseen to improve the time resoultion and the fill factor and to study the radiation hardness of the devices.

\section{Acknowledgments}

The author acknowledge the fundamental contributions coming from the RD50, UFSD and the APIX2 collaborations and specifically the following colleagues for all the useful discussions and for providing with material for this presentation: N. Cartiglia, L. Pancheri, R. Arcidiacono, G. Dalla Betta, M. Ferrero, M. Mandurrino, V. Sola, A. Staiano. Part of the presented work has been financed by the European Union's Horizon 2020 Research and Innovation funding program, under Grant Agreement no. 654168 (AIDA-2020) and Grant Agreement no. 669529 (ERC UFSD669529), by the Italian Ministero degli Affari Esteri and INFN Gruppo V. The author is partially supported by the project Ricerca locale 2016 OBEM_RILO_16_01 (Studio del ruolo delle cellule parenchimatiche nel recupero di embolismi).

\section{References}

[1] G. Apollinari et al., High-Luminosity Large Hadron Collider (HL-LHC): Preliminary Design Report, CERN-2015-005 (2015) 
[2] R. Yohay, Precision timing for the High Luminosity Upgrade of CMS, in this Proceedings

[3] G. Kramberger, High Granularity Timing Detector at Atlas, in this Proceedings

[4] The LHCb collaboration, Expression of Interest for a Phase-II LHCb Upgrade, CERN-LHCC-2017-003 (2017)

[5] E. Migliore, 4th dimensional tracking: the GigaTracker of NA62 experiment, in this Proceedings

[6] H. Sadrozinski, A. Seiden, N. Cartiglia, 4-Dimensional tracking with Ultra-Fast Silicon Detectors, Reports of Progress in Physics, ROPP-100788.R2

[7] L. Pancheri, et al., First prototypes of two-tier avalanche pixel sensors for particle detection, Nucl. Instrum. Meth. A845 (2017) 143

[8] L. Pancheri, State of the art and perspectives of CMOS avalanche detectors, CERN seminar (2017)

[9] S. Parker et al., Increased speed: 3D silicon sensors; fast current amplifiers, IEEE Trans. Nucl. Sci. NS-58, 2 (2011) 404

[10] G. Pellegrini, et al., Technology developments and first measurements of Low Gain Avalanche Detectors (LGAD) for high energy physics applications, Nucl. Instrum. Meth. A765 (2014) 24

[11] RD50 collaboration, http://rd50.web.cern.ch/rd50

[12] H.Sadrozinski et al., Timing Measurements on Ultra-Fast Silicon Detectors, 12th Trento Workshop on Advanced Silicon Radiation Detectors (2017) Trento, Italy

[13] N. Cartiglia, et al., Beam test results of a 16 ps timing system based on ultra-fast silicon detectors, Nucl. Instrum. Meth. A850 (2017) 83

[14] F. Ravera, et al., The CT-PPS project: detector hardware and operational experience, in this Proceedings

[15] J. Lange, et al., Gain and time resolution of $45 \mu \mathrm{m}$ thin Low Gain Avalanche Detectors before and after irradiation up to a fluence of $10^{15} \mathrm{n}_{e q} / \mathrm{cm}^{2}$, JINST 12 (2017) P05003

[16] Z. Galloway, et al., Properties of HPK UFSD after neutron irradiation up to $6 \cdot 10^{15} \mathrm{n}_{e q} / \mathrm{cm}^{2}$, arxiv:1707.04961

[17] N. Cartiglia, et al., Tracking in 4 dimensions, PoS (EPS-HEP2017) 489 (2017)

[18] L. Pancheri, et al., First demonstration of a two-tier pixelated avalanche sensor for charged particle detection, IEEE Journal of the Electron Devices Society 5 (2017) 404;

[19] N. D’Ascenzo, et al., Silicon avalanche pixel sensor for high precision tracking, JINST 9 (2014) C03027;

[20] M. Perenzoni, et al., Compact SPAD-Based Pixel Architectures for Time-Resolved Image Sensors, Sensors 16 (2016) 745;

[21] A. Ficorella, et al., Crosstalk mapping in CMOS SPAD arrays, 46th European Solid-State Device Research Conference (ESSDERC) (2016) 101 